\title{
Performance of Relay-Aided DS-CDMA Downlink Systems Communicating over Nakagami-m Fading Channels
}

\author{
Wei Fang, Lie-Liang Yang and Lajos Hanzo \\ School of ECS, University of Southampton \\ SO17 1BJ, United Kingdom \\ Tel: +44-23-8059 3364, Fax: +44-23-8059 4508 \\ E-mail:wf04r,lly,lh@ecs.soton.ac.uk, http://www-mobile.ecs.soton.ac.uk
}

\begin{abstract}
In this contribution we investigate the bit-error-rate (BER) performance of a downlink direct-sequence code-division multiple-access (DS-CDMA) system, where a set of relays are chosen to assist a mobile terminal (MT) in order to improve its communications reliability with the base-station (BS). In our relay-aided DS-CDMA system, the downlink signal is detected at the relays based on the minimum mean-square-error (MMSE) principles, since the MMSE detector can be implemented with the aid of low-complexity adaptive techniques. At the destination MTs (DMTs) the signals are combined according to the maximum signal-to-interference-plus-noise ratio (MSINR) criterion. The BER performance of the relay-aided DS-CDMA downlink is investigated, when the communications channels are assumed to experience both propagation pathloss and fast Nakagami- $m$ fading. Furthermore, we consider the impact of the relays' locations and that of the power-allocation among the BS and relays on the achievable BER performance. From our study and simulation results, it can be shown that full relay-diversity gain can be achieved, after the multiuser interference (MUI) and inter-relay interference (IRI) are suppressed by the MMSE and MSINR detectors.
\end{abstract}

\section{INTRODUCTION}

In wireless communications spatial diversity is attractive due to its capability of offering spectral-efficiency without incurring an expenditure of transmission time and bandwidth [1]. Spatial diversity can usually be achieved with the assistance of deploying multiple transmit antennas and/or multiple receive antennas [2]. However, it is usually impractical for a MT to employ multiple antennas due to the size or cost limitation. For this sake, cooperative diversity [3-5] has been proposed, which is capable of achieving the spatial diversity without using multiple antennas at each MT. Instead, it takes advantage of the broadcast nature of wireless channels and transmits a message both directly and via relays to its DMT. This type of cooperative diversity is usually referred to as relay diversity.

It is well-known that, for downlink transmission, transmit diversity can be implemented by deployment of multiple transmit antennas at BS [6]. While employing multiple antennas solely at the BS is capable of achieving the transmit diversity, it does not exploit the non-linear relation between the propagation pathloss of radio signals and the propagation distance from the BS to a MT. By contrast, in relay-aided communications, an intermediate relay between the BS and MT splits a longer communication path into two shorter segments. Thus, the overall pathloss may be reduced by exploiting the non-linear relation between the propagation pathloss and propagation distance, which allows potentially reduction of overall transmission power. Furthermore, if a relay is chosen in the vicinity of the DMT, it may need considerably lower power for forwarding information to the DMT. In this case, the total transmission power for the DS-CDMA downlink may be allocated in such a way that the minimum possible BER can be achieved.

In this contribution we investigate the BER performance of the DS-CDMA downlink, when one DMT is aided by a cluster of other MTs acting as relays, in order to achieve the relay diversity and to mitigate the impact of propagation pathloss. In our system MMSE detection is adopted by the relays for mitigating the MUI. Finally, the information at the DMTs are detected based on the MSINR criterion. Note that, although both the MMSE and MSINR detectors achieve the same BER performance, the MMSE detection is employed by the relays, since the MMSE detection uses directly the auto-correlation matrix of the received observation, which is easy to estimate in practice. In our investigation both the propagation pathloss and fast fading [7] are considered, in order to demonstrate the fact that appropriately selected relays can be beneficial to saving the transmission power and hence achieving a higher powerefficiency. In this contribution, the fast fading is modelled by the generalized Nakagami- $m$ fading [8], where signals transmitted from the BS to the relays and that from the relays to the DMT may experience different fading. Our analysis and simulation results show that the relays of a given DMT should be chosen from certain geographical area, in order to achieve the best available BER performance. Furthermore, it can be shown that the full diversity gain can be achieved, when optimum powerallocation among the direct and relay channels is utilized; the BER performance of the DS-CDMA downlink improves when the number of relays increases.

\section{SySTEM DESCRIPTION}

\section{A. Transmitted Signal}

In our considered downlink DS-CDMA system, the BS broadcasts the sum of $K$ downlink signals to the $K$ DMTs. We assume that there are $L$ relays in the vicinity of each of the $K$ DMTs, as shown in Fig. 1. The DS-CDMA signal transmitted at the BS can be expressed as

$$
x(t)=\sum_{k=1}^{K} x_{k}(t)
$$

where $x_{k}(t)$ represents the signal transmitted to the $k$ th MT, which can be expressed as

$$
x_{k}(t)=\sqrt{2 P_{k t}} b_{k}(t) c_{k}(t) \cos \left(2 \pi f_{c} t+\phi_{k}\right)
$$




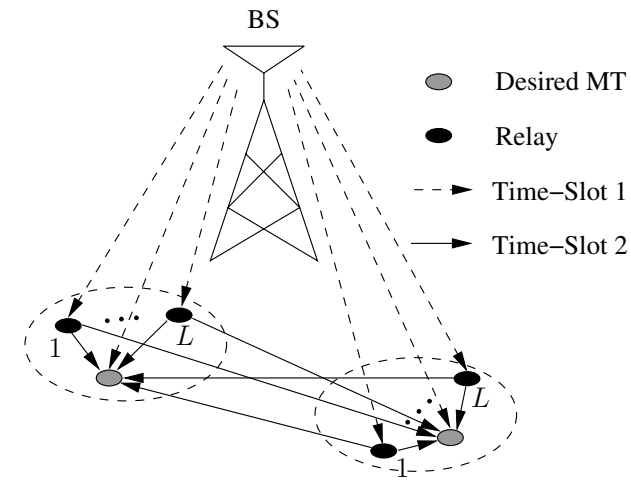

Fig. 1. Schematic block diagram for the relay-aided DS-CDMA downlink supporting multiple users.

where $P_{k t}$ represents the transmission power corresponding to the $k$ th $\mathrm{MT}, f_{c}$ is the carrier frequency and $\phi_{k}$ denotes the initial phase angle associated with the carrier modulation. In (2) $b_{k}(t)$ represents the transmitted data waveform, which can be represented as

$$
b_{k}(t)=\sum_{n=0}^{\infty} b_{k}[n] P_{T_{b}}\left(t-n T_{b}\right)
$$

where $b_{k}[n] \in\{-1,+1\}, T_{b}$ represents the bit duration, $P_{T_{b}}(t)$ represents the rectangular waveform, which is defined as $P_{T_{b}}(t)=1$ if $0 \leq t<T_{b}$, and $P_{T_{b}}(t)=0$ otherwise. Furthermore, in (2), $c_{k}(t)$ represents the DS spreading waveform, which can be denoted as

$$
c_{k}(t)=\sum_{n=0}^{\infty} c_{k n} \psi_{T_{c}}\left(t-n T_{c}\right)
$$

where $T_{c}$ represents the chip-duration, $N=T_{b} / T_{c}$ represents the spreading factor, $c_{k n} \in\{-1,+1\}, \psi_{T_{c}}(t)$ is the chipwaveform, which is defined within $\left[0, T_{c}\right)$ and normalized to satisfy $\int_{0}^{T_{c}} \psi_{T_{c}}^{2}(t) d t=T_{c}$. Let us now describe the cooperation scheme.

\section{B. Cooperation Operation}

As shown in Fig. 1, we assume that each DMT is aided by $L$ relays for signal reception from the BS. The relays are constituted by the MTs, which do not have their own information to communicate. We assume that the relays for a DMT are generally selected in the vicinity of the DMT in order to improve the power-efficiency of the relay-aided DS-CDMA downlink, which will become explicit in our forthcoming discourse. For convenience of description, we define the direct channels (Dchannels) as the channels connecting the BS with the DMTs, the relay channels (R-channels) as the channels connecting the BS through relays to the DMTs. Furthermore, a R-channel is further divided into the BR-channel connecting the BS to a relay and the RM-channel connecting the relay to the DMT.

We assume that the proposed cooperation scheme is operated based on time-division (TD). Specifically, we divide a symbol duration $T_{s}$ into two consecutive time-slots, both of which have a duration of $T_{b}$. In the considered cooperation scheme, the BS broadcasts the signal in the form of (1) to the $K$ DMTs and the $K L$ relays using the first time-slot. Within the second time-slot, the $K L$ relays forward their signals received from the BS in the first time-slot to the $K$ DMTs. Then, the complex baseband equivalent signal received by the $l$ th relay of the $k$ th DMT within the first time-slot of the $n$th symbol duration can be expressed as

$$
\begin{array}{r}
r_{l}^{(k)}(t)=h_{l}^{(k)} \sum_{k^{\prime}=1}^{K} \sqrt{2 P_{k^{\prime}, l}^{(k)}} b_{k^{\prime}}[n] c_{k^{\prime}}(t)+n_{l}^{(k)}(t), \\
k=1,2, \cdots, K ; l=1,2, \cdots, L
\end{array}
$$

where $P_{k^{\prime}, l}^{(k)}$ represents the power received at the $l$ th relay of the $k$ th DMT after taking into account the propagation pathloss, $h_{l}^{(k)}$ denotes the (fast) fading gain of the $l$ th BR-channel connecting the BS with the $l$ th relay of the $k$ th DMT, while $n_{l}^{(k)}(t)$ represents the complex baseband equivalent Gaussian noise observed at the $l$ th relay of the $k$ th DMT, $n_{l}^{(k)}(t)$ is assumed to be a Gaussian process with mean zero and a single-sided power spectral density of $N_{0}$ per dimension.

At the $l$ th relay of the $k$ th DMT, $r_{l}^{(k)}(t)$ is first input to a filter matched to the chip-waveform $\psi_{T_{c}}(t)$. Then, the matchedfilter's (MF's) output is sampled at the chip-rate, which provides $N$ samples per bit to the relay for detection. At the relays, the signals transmitted to the DMTs are detected based on the MMSE principles, in order to mitigate the effect of both the MUI and thermal noise. In this contribution we assume that each relay has the knowledge of its BR-channel and also the spreading sequence of the DMT it serves, but no knowledge of the other interfering DMTs. Let us define

$$
\begin{aligned}
\boldsymbol{y}_{l}^{(k)} & =\left[y_{l 0}^{(k)}, y_{l 1}^{(k)}, \cdots, y_{l(N-1)}^{(k)}\right]^{T} \\
\boldsymbol{n}_{l}^{(k)} & =\left[n_{l 0}^{(k)}, n_{l 1}^{(k)}, \cdots, n_{l(N-1)}^{(k)}\right]^{T} \\
\boldsymbol{c}_{k} & =\frac{1}{\sqrt{N}}\left[c_{k 0}, c_{k 1}, \ldots, c_{k(N-1)}\right]^{T}
\end{aligned}
$$

Then, it can be shown that $\boldsymbol{y}_{l}^{(k)}$ corresponding to the $l$ th relay of the $k$ the DMT can be expressed as

$$
\boldsymbol{y}_{l}^{(k)}=c_{k} h_{l}^{(k)} b_{k}[n]+\underbrace{h_{l}^{(k)} \sum_{k^{\prime} \neq k}^{K} \sqrt{\frac{P_{k^{\prime}, l}^{(k)}}{P_{k, l}^{(k)}} \boldsymbol{c}_{k^{\prime}} b_{k^{\prime}}[n]+\boldsymbol{n}_{l}^{(k)}}}_{\boldsymbol{I}_{k l}}
$$

where $\boldsymbol{n}_{l}^{(k)}$ is a $N$-length noise vector obeying the Gaussian distribution associated with mean zero and a covariance matrix of $N_{0} / E_{l}^{(k)} \boldsymbol{I}_{N}$, where $E_{l}^{(k)}=P_{k, l}^{(k)} T_{b}$ denotes the energy per bit received by the $l$ th relay of the $k$ th DMT from the BS and $I_{N}$ is a $N$-dimensional identity matrix.

Based on $\boldsymbol{y}_{l}^{(k)}$, the $l$ th relay of the $k$ th DMT processes it with a complex weight vector $\boldsymbol{w}_{k l}$ of length $N$ in MMSE principles, yielding the estimate $\hat{b}_{l}^{(k)}[n]$ to $b_{k}[n]$

$$
\hat{b}_{l}^{(k)}[n]=\left(\boldsymbol{w}_{k l}^{o p t}\right)^{H} \boldsymbol{y}_{l}^{(k)}
$$

where $\boldsymbol{w}_{k l}^{o p t}$ is given by

$$
\boldsymbol{w}_{k l}^{o p t}=\boldsymbol{R}_{y_{l}^{(k)}}^{-1} \boldsymbol{r}_{y_{l}^{(k)} b_{k}}
$$

where $\boldsymbol{R}_{y_{l}^{(k)}}$ is the auto-correlation matrix of the observation vector $\boldsymbol{y}_{l}^{(k)}$ of (7), which can be estimated from the received 
observations as

$$
\boldsymbol{R}_{y_{l}^{(k)}}=E\left[\boldsymbol{y}_{l}^{(k)}\left(\boldsymbol{y}_{l}^{(k)}\right)^{H}\right]
$$

while $\boldsymbol{r}_{y_{l}^{(k)} b_{k}}$ in (9) represents the cross-correlation matrix between the observation vector $\boldsymbol{y}_{l}^{(k)}$ and the desired bit $b_{k}[n]$, which is given by

$$
\boldsymbol{r}_{y_{l}^{(k)} b_{k}}=\mathrm{E}\left[\boldsymbol{y}_{l}^{(k)} b_{k}[n]\right]=\boldsymbol{c}_{k} h_{l}^{(k)}
$$

Furthermore, it can be shown that the estimate $\hat{b}_{l}^{(k)}[n]$ can be simply expressed as

$$
\hat{b}_{l}^{(k)}[n]=\eta_{k l} b_{k}[n]+n_{k l}
$$

by defining $\eta_{k l}=\left(\boldsymbol{c}_{k} h_{l}^{(k)}\right)^{H} \boldsymbol{R}_{y_{l}^{(k)}}^{-1} \boldsymbol{c}_{k} h_{l}^{(k)}$ and $n_{k l}=$ $\left(\boldsymbol{c}_{k} h_{l}^{(k)}\right)^{H} \boldsymbol{R}_{y_{l}^{(k)}}^{-1} \boldsymbol{I}_{k l}$.

After the detection, $\hat{b}_{l}^{(k)}[n]$ is then re-spread and forwarded by the $l$ th relay of the $k$ th DMT to the $k$ th DMT. Correspondingly, the transmitted signal of the $l$ th relay of the $k$ th DMT can be expressed by

$$
\begin{array}{r}
s_{l}^{(k)}(t)=\sqrt{2 P_{l t}^{(k)}} \beta_{l}^{(k)} \hat{b}_{l}^{(k)}[n] c_{l}^{(k)}(t) \cos \left(2 \pi f_{c} t+\phi_{l}^{(k)}\right), \\
k=1,2, \cdots, K ; l=1,2, \cdots, L
\end{array}
$$

where $P_{l t}^{(k)}, c_{l}^{(k)}(t)$ and $\phi_{l}^{(k)}$ represent, respectively, the transmission power, signature waveform and initial phase. $c_{l}^{(k)}(t)$ can be expressed as

$$
c_{l}^{(k)}(t)=\sum_{n=0}^{\infty} c_{l n}^{(k)} \psi_{T_{c}}\left(t-n T_{c}\right)
$$

where $c_{l n}^{(k)} \in\{-1,+1\}$. Furthermore, in (13), $\beta_{l}^{(k)}$ is a normalization coefficient for $s_{l}^{(k)}(t)$, which is given by

$$
\beta_{l}^{(k)}=\sqrt{1 /\left(\boldsymbol{w}_{k l}^{H} \boldsymbol{R}_{y_{l}^{(k)}} \boldsymbol{w}_{k l}\right)}
$$

\section{Channel Modelling}

The channel model considered includes both propagation pathloss and fast fading. We assume that the propagation pathloss satisfies the $n$th power law and the pathloss, $L_{p}(d)$, as a function of transmitter-receiver (T-R) separation distance $d$, can be expressed as [7]

$$
L_{p}(d)(d B)=L_{s}\left(d_{0}\right)(d B)+10 n \log \left(d / d_{0}\right)
$$

where, $L_{s}\left(d_{0}\right)$ is the pathloss measured at the reference distance $d_{0}, n$ is the pathloss exponent, which takes a typical value of 2 in free-space and 4 in cellular mobile systems.

In the context of the fast fading of the D-channels, BRchannels and the RM-channels, we assume a generalized Nakagami- $m$ fading channel model. For convenience, we drop the index of $k$ and $k^{\prime}$, and let in (5) $h_{l}^{(k)}=\left|h_{l 1}\right| e^{j \theta_{l 1}}$ and in (19) $h_{k, r l}^{\left(k^{\prime}\right)}=\left|h_{l 2}\right| e^{j \theta_{l 2}}$, where $\left|h_{l 1}\right|,\left|h_{l 2}\right|$ and $\theta_{l 1}, \theta_{l 1}$ denote the amplitudes and phases of the BR-channels and RM-channels, respectively. Then, with the assumption of Nakagami- $m$ fading, the probability density functions (PDF) of $\left|h_{l i}\right|, i=1,2$, can be expressed as $[8,10]$

$$
f_{\left|h_{l i}\right|}(y)=\frac{2 m_{l i}^{m_{l i}} y^{2 m_{l i}-1}}{\Gamma\left(m_{l i}\right) \Omega_{l i}} e^{-\left(m_{l i} y^{2} / \Omega_{l i}\right)}, i=1,2
$$

where $\Gamma(\cdot)$ is the gamma function $[11], \Omega_{l i}=E\left[\left|h_{l i}\right|^{2}\right]$ and $m_{l i}$ represents the fading parameter corresponding to the BRchannel when $i=1$ or to the RM-channel when $i=2$. Note that $h_{0}^{(k)}=\left|h_{0}\right| e^{j \theta_{0}}$, where $\left|h_{0}\right|$ and $\theta_{0}$ signify the amplitude and phase of the D-channel. The PDF of $h_{0}$ can be readily obtained from (17) with $m_{l i}$ replaced by $m_{0}$ and $\Omega_{l i}$ by $\Omega_{0}$. Furthermore, the phases $\theta_{0}$ and $\theta_{l i}$ are assumed to be the independent identically distributed (iid) random variables uniformly distributed within $[0,2 \pi)$.

\section{RECEIVED SignAL AT THE DMTS}

At the $k$ th DMT, the received baseband equivalent signal from the first time-slot of the $n$th symbol duration can be expressed as

$$
r_{0}^{(k)}(t)=h_{0}^{(k)} \sum_{k=1}^{K} \sqrt{2 P_{k r}} b_{k}[n] c_{k}(t)+n(t)
$$

where $P_{k r}$ denotes the power received by the $k$ th DMT from the BS after taking into account of the propagation pathloss, $h_{0}^{(k)}$ represents the (fast) channel gain of the $k$ th D-channel, while $n(t)$ is the Gaussian random process associated with zero mean and a single-sided power spectral density of $N_{0}$ per dimension. By contrast, the complex baseband equivalent signal received by the $k$ th MT within the second time-slot of the $n$th symbol duration can be expressed as

$$
r_{1}^{(k)}(t)=\sum_{k^{\prime}=1}^{K} \sum_{l=1}^{L} \sqrt{2 P_{k, l r}^{\left(k^{\prime}\right)}} h_{k, r l}^{\left(k^{\prime}\right)} \beta_{l}^{\left(k^{\prime}\right)} \hat{b}_{l}^{\left(k^{\prime}\right)}[n] c_{l}^{\left(k^{\prime}\right)}(t)+n(t)
$$

where $P_{k, l r}^{\left(k^{\prime}\right)}$ represents the power received by the $k$ th DMT from its $l$ th relay, again, after taking into account of the propagation pathloss, and $h_{k, r l}^{\left(k^{\prime}\right)}$ represents the (fast) channel gain of the $l$ th RM-channel of DMT $k^{\prime}$.

The received signal at the DMT $k$ is first filtered by a chipwaveform MF and then sampled at the chip-rate in order to generate observation samples. Since the signals to the DMTs are transmitted within two time-slots, each of which is associated with the spreading sequences of length $N$, hence, the $k$ th DMT can obtain a total of $2 N$ observation samples for detecting $b_{k}[n]$. Without loss of generality, we assume that the first DMT is the reference DMT to be detected. Let $\boldsymbol{y}=\left[\boldsymbol{y}_{0}^{T}, \boldsymbol{y}_{1}^{T}\right]^{T}$ contain the $2 N$ observation samples, where $\boldsymbol{y}_{i}=\left[y_{i 0}, y_{i 1}, \cdots, y_{i(N-1)}\right]^{T}, i=0,1$. Then, it can be shown that $\boldsymbol{y}$ can be expressed as

$$
\boldsymbol{y}=\boldsymbol{C} \boldsymbol{h}_{1} b_{1}[n]+\underbrace{\boldsymbol{n}_{I}+\tilde{\boldsymbol{n}}}_{\tilde{\boldsymbol{n}}_{I}}
$$

where $C$ is a $2 N \times(L+1)$ matrix constructed as

$$
\boldsymbol{C}=\left[\begin{array}{ccccc}
\boldsymbol{c}_{1} & \mathbf{0} & \mathbf{0} & \cdots & \mathbf{0} \\
\mathbf{0} & \boldsymbol{c}_{1}^{(1)} & \boldsymbol{c}_{2}^{(1)} & \cdots & \boldsymbol{c}_{L}^{(1)}
\end{array}\right]
$$

associated with $\boldsymbol{c}_{l}^{(k)}=\left[c_{l 0}^{(k)}, c_{l 1}^{(k)}, \cdots, c_{l(N-1)}^{(k)}\right] / \sqrt{N}$, $h_{1}$ is formed by the normalized channel gains 
of the reference DMT, which is given by $\boldsymbol{h}_{1}=$ $\left[h_{0}^{(1)}, \sqrt{\zeta_{11}} h_{1, r 1}^{(1)} \eta_{11}, \sqrt{\zeta_{12}} h_{1, r 2}^{(1)} \eta_{12}, \cdots, \sqrt{\zeta_{1 L}} h_{1, r L}^{(1)} \eta_{1 L}\right]^{T}$, where $\zeta_{k l}=\left(\beta_{l}^{(k)}\right)^{2} P_{1, l r}^{(k)} / P_{1 r}$. Finally, in (21) $n_{I}$ contains the interference and $\tilde{\boldsymbol{n}}=\left[\tilde{\boldsymbol{n}}_{0}^{T}, \tilde{\boldsymbol{n}}_{1}^{T}\right]^{T}$ is a $2 N$-length vector, where $\tilde{\boldsymbol{n}}_{i}, i=0,1$, obeys the Gaussian distribution with mean zero and a covariance matrix of $\left(N_{0} / E_{1 r}\right) \boldsymbol{I}_{N}$, where $E_{1 r}=P_{1 r} T_{b}$ denotes the average energy per bit received by the first DMT from the BS during the first time-slot.

\section{Maximum SinR Assisted Multiuser Combining}

The signals containing the information of the reference DMT are combined based on the MSINR principles [12]. Specifically, let us re-write the observation vector of (20) as

$$
\boldsymbol{y}=\boldsymbol{C h}_{1} b_{1}[n]+\tilde{\boldsymbol{n}}_{I}
$$

Then, according to the MSINR principles, it can be shown that the optimum weight vector can be expressed by [12]

$$
\boldsymbol{w}_{o p t}=\mu \boldsymbol{R}_{I}^{-1} \boldsymbol{C h} \boldsymbol{h}_{1}
$$

where $\mu>0$ is a constant and $\boldsymbol{R}_{I}=E\left[\tilde{\boldsymbol{n}}_{I} \tilde{\boldsymbol{n}}_{I}^{H}\right]$ is the covariance matrix of $\tilde{\boldsymbol{n}}_{I}$. Correspondingly, the decision variable for the reference DMT can be denoted as $z_{1}=\Re\left\{\boldsymbol{w}_{o p t}^{H} \boldsymbol{y}\right\}$, where $\Re\{x\}$ represents the real-part of $x$.

\section{Performance Results}

In this section we provide a range of simulation results in order to illustrate the BER performance for the relay-aided DSCDMA downlink systems, when the MMSE multiuser detector (MUD) is adopted by the relays and the MSINR MUD is employed by the DMTs. Specifically, in our simulations we assume that the binary phase-shift keying (BPSK) baseband modulation scheme is employed. In this contribution, in order to carry out a fair comparison, we assume that the total tranmission power consumed per symbol is the same for both our proposed relay-aided system and the conventional system, which does not use relays, i.e., we assume that $P_{0}=P_{k t}+L P_{l t}^{(k)}$, where $P_{0}$ is the total power radiated in the context of one DMT in the conventional system. In our proposed relay-aided system, $\alpha P_{0}$ for a DMT is allocated to the first time-slot for the BS to transmit signals to the DMT and its relays. By contrast, the amount of $(1-\alpha) P_{0}$ for a DMT is allocated to the second time-slot for the relays of a DMT to forward the information to the DMT. In our simulations all the distances in the system are normalized by the distance $d_{B M}$ between the BS and the reference DMT. We assume that the BS and the reference DMT are located at $(1,0)$ and $(0,0)$, respectively, while the cluster of relays of the reference DMT is located at $(\delta, 0)$, where $0<\delta<1$. Furthermore, for the sake of simplicity, we assume that the power-allocations for all the DMTs are the same, so are the location parameters of their relays.

In Fig. 2, we evaluate the BER versus power-allocation $(\alpha)$ and relays' location $(\delta)$ performance of the relay-aided DSCDMA downlink system supporting single user, when the Dchannel and BR-channels experience Rayleigh fading, while the RM-channels experience Nakagami- $m$ fading associated with $m_{l 2}=2$ for $L=3$. In our simulations we assumed that the pathloss exponent corresponding to the large-scale-fading was $n=4$ and the SNR per bit was $E_{b} / N_{0}=4 \mathrm{~dB}$. It can be seen from Fig. 2 that the BER performance improves when assigning more power to the first time slot. Furthermore, when increasing the value of $\delta$, implying that the relays move from the DMT toward the BS, the BER performance first improves and then degrades. Therefore, as seen in Fig. 2, for any a given value of $\alpha$, there may exist an optimum value of $\delta$, which results in the lowest BER. Vice versa, for any a given value of $\delta$, there exists an optimum value of $\alpha$, implying the optimum powerallocation, which also results in the lowest BER. Specifically, the optimum $(\alpha, \delta)$ values are approximately $\alpha=0.8, \delta=0.4$ for $n=3$ as shown in Fig. 2. Our study in [13,14] for DSCDMA uplink also shows that the optimum $(\alpha, \delta)$ values are approximately $\alpha=0.9, \delta=0.3$ for $n=4$.

In Figs. 3 and 4 we evaluate the BER versus the average SNR per bit performance of the relay-aided DS-CDMA downlink supporting $K=2$ users, when the D-channel and BRchannels experience Rayleigh fading, while the RM-channels experience Nakagami- $m$ fading associated with $m_{l 2}=2$. In our simulations we assumed that the $m$-sequences of length 15 were employed. The other parameters for Fig. 3 were $\alpha=0.8$, $\delta=0.4$ and $n=3$, while those for Fig. 4 were $\alpha=0.9$, $\delta=0.3$ and $n=4$. From the results of Figs. 3 and 4, it can be observed that, when the optimum power-allocation is considered, the BER performance of the DS-CDMA downlink significantly improves, when the DMTs are aided by more relays, yielding a higher relay diversity.

In Fig. 5, we investigate the BER performance of the relayaided DS-CDMA downlink, when the random sequences of length 15 are employed instead of the $m$-sequences in Figs. 3 and 4. As shown in Fig. 5, two different pathloss exponents were considered, which were $n=4$ for the case of $\alpha=0.9$, $\delta=0.3$ and $n=3$ for the case of $\alpha=0.8, \delta=0.4$. In comparison with the results in Figs. 3 and 4, it can be observed that the BER performance of using the $m$-sequences is only slightly better than that of using the random sequences, when assuming the same number of relays and the same fading environment. This observation implies that the MSINR MUD is capable of mitigating efficiently the interference from the relays. From the results of Figs. 3-5, it can be seen that the performance corresponding to $n=3$ is $3.0-3.7 \mathrm{~dB}$ worse than that corresponding to $n=4$ at a BER of $10^{-5}$. This observation implies that the relay-aided DS-CDMA downlink is capable of achieving a better BER performance in an environment with severe propagation pathloss than in an environment experiencing less severe propagation pathloss. Therefore, the relay-aided DSCDMA downlink is capable of reducing the overall propagation pathloss by exploiting the non-linear relation between the propagation pathloss and propagation distance. In contributions, we have proposed a relay diversity scheme for the DS-CDMA downlink transmission, where each of the DMTs is aided by a set of relays. The BER performance of the relay-aided DSCDMA downlink has been investigated, when the MMSEand MSINR-aided MUDs are employed and when assuming that the DS-CDMA downlink signals experience Nakagami$m$ fast fading associated with propagation pathloss. Our study and simulation results show that the minimum achievable BER performance of the relay-aided DS-CDMA downlink depends on a range of system parameters, including locations of the relays, power-allocation between the first and second timeslots, pathloss exponent, etc. It can be shown that the BER performance of the relay-aided DS-CDMA downlink can be significantly enhanced, when optimum power-allocation is applied and when more relays per DMT are employed. 


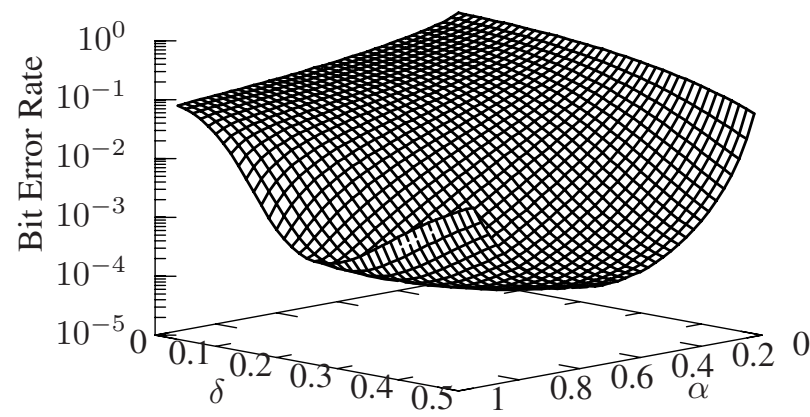

Fig. 2. BER versus power-allocation $(\alpha)$ and relays' location $(\delta)$ performance for the DS-CDMA downlink using relay diversity and $m$-sequences, when the D-channel and BR-channels experience Rayleigh fading, while the RMchannels experience Nakagami- $m$ fading associated with $m_{l 2}=2$ and the pathloss exponent is $n=4$. The other parameters were $E_{b} / N_{0}=4 \mathrm{~dB}$ and $L=3$.

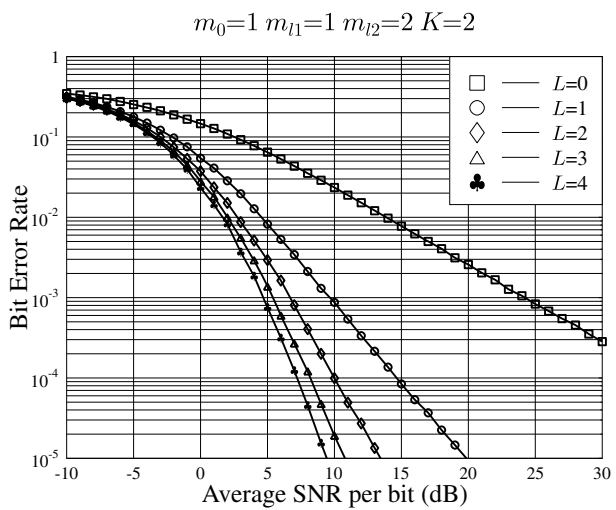

Fig. 3. BER versus SNR per bit performance for the relay-aided DSCDMA downlink, when the D-channel and BR-channels experience Rayleigh fading, while the RM-channels experience Nakagami- $m$ fading associated with $m_{l 2}=2$ for $L=1,2,3,4$. In our simulations, $m$-sequences of length 15 were employed and the other parameters were $\alpha=0.8, \delta=0.4$ and $n=3$.

\section{ACKNOWLEDGEMENT}

The authors would like to acknowledge with thanks the financial assistance from EPSRC of UK.

\section{REFERENCES}

[1] R. U. Nabar and H. Bölcskei, "Space-time signal design for fading relay channels," in Proc. IEEE Globecom, vol. 4, pp. 1952-1956, Dec. 2003.

[2] A. Goldsmith, S. A. Jafar, N. Jindal, and S. Vishwanath, "Capacity limits of MIMO channels," IEEE Journal on Selected Areas in Communications, vol. 21, pp. 684-702, June 2003.

[3] A. Sendonaris, et.al, "User cooperation diversity-Part I and II," IEEE Trans. on Commun., vol. 51, pp. 1927-1938, Nov. 2003.

[4] J. N. Laneman, D. N. C. Tse, and G. W. Wornell, "Cooperative diversity in wireless networks: Efficient protocols and outage behavior," IEEE Trans. on Information Theory, vol. 50, pp. 3062-3080, December 2004.

[5] A. Bletsas, A. Khisti, D. Reed, and A. Lippman, "A simple cooperative diversity method based on network path selection," IEEE Journal on Selected Areas in Communications, vol. 24, no. 3, pp. 659-672, 2006.

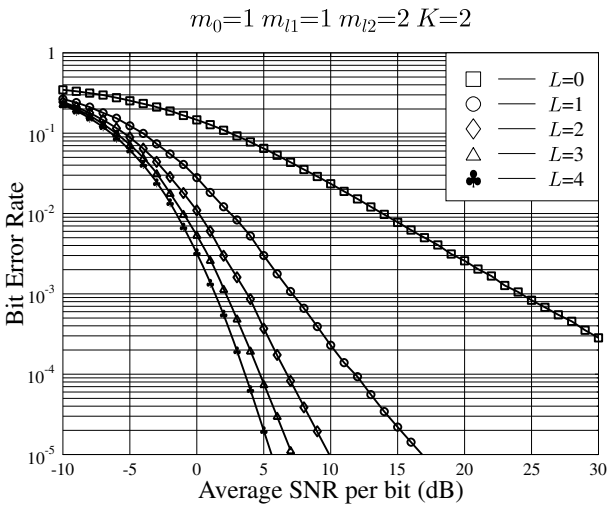

Fig. 4. BER versus SNR per bit performance for the relay-aided DS-CDMA downlink, when the D-channel and BR-channels experience Rayleigh fading, while the RM-channels experience Nakagami- $m$ fading associated with $m_{l 2}=$ 2 for $L=1,2,3,4$. In our simulations, $m$-sequences were employed and the other parameters were $\alpha=0.9, \delta=0.3$ and $n=4$.

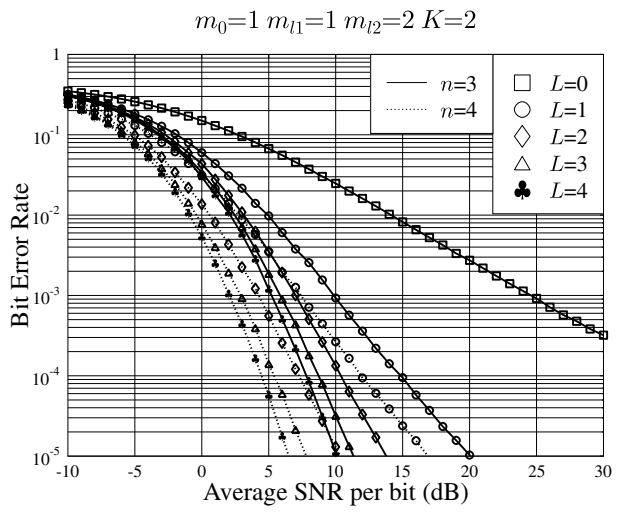

Fig. 5. BER versus SNR per bit performance for relay-aided DS-CDMA downlink, when the D-channel and BR-channels experience Rayleigh fading, while the RM-channels experience Nakagami- $m$ fading associated with $m_{l 2}=2$ for $L=1,2,3,4$. In our simulations, random sequences were used and two different pathloss exponents were considered, namely, $n=4$ associated with $\alpha=0.9, \delta=0.3$ and $n=3$ associated with $\alpha=0.8, \delta=0.4$.

[6] F. Rashid-Farrokhi, K. J. R. Liu, and L. Tassiulas, "Transmit beamforming and power control for cellular wireless systems," IEEE Journal on Selected Areas in Communications, vol. 16, pp. 1437-1450, Oct. 1998.

[7] T. S. Rappaport, Wireless Communications: Principles and Practice Upper Saddle River, NJ: Prentice Hall, 1996.

[8] N. Nakagami, "the $m$-distribution, a general formula for intensity distribution of rapid fading," in Statistical Methods in Radio Wave Propagation (W. G. Hoffman, ed.), Oxford, England: Pergamon, 1960.

[9] S. Haykin, Adaptive Filter Theory. New Jersey: Prentice Hall, 1996.

[10] L.-L. Yang and L. Hanzo, "Performance of generalized multicarrier DS-CDMA over Nakagami- $m$ fading channels," IEEE Transactions on Communications, vol. 50, pp. 956 - 966, June 2002.

[11] J. G. Proakis, Digital Communications. New York: McGraw-Hill, 1995.

[12] Compton, R. T. (Jr), Adaptive Antennas: Concepts and Performance. Englewood Cliffs, New Jersey, USA: Prentice-Hall, 1996.

[13] W. Fang, L.-L. Yang, and L. Hanzo, "Single-user performance of DSCDMA using relay diversity and power allocation," To appear in IET Proceedings Communications, 2008.

[14] W. Fang, L.-L. Yang, and L. Hanzo, "Single-user performance of relayaided DS-CDMA with power allocation and inter-relay interference suppression," in IEEE VTC2007-Fall, (Baltimore), 30 September - 3 October 2007. 